\title{
Lidocaína com vasoconstrictor isolada e associada ao fentanil via peridural em cães
}

\author{
Lidocaine with vasoconstrictor isolated and its combination with epidural fentanyl anesthesia in dogs
}

\author{
Renata Navarro Cassu ${ }^{{ }^{*}}$ Alessandra Melchert ${ }^{\mathrm{II}}$ Ana Paula Gasparotto da Silva ${ }^{\mathrm{III}}$ \\ Atilana Maniezo dos Reis ${ }^{\text {III }}$ Carlos Collares Meirelles ${ }^{\mathrm{I}}$
}

\section{RESUMO}

O objetivo deste estudo cego foi avaliar os efeitos cardiorrespiratórios e analgésicos de diferentes doses de fentanil associado à lidocaína com vasoconstrictor via peridural em cães. Foram avaliados 28 cães adultos, distribuídos em quatro tratamentos: $5 \mathrm{mg} \mathrm{kg}^{-1}$ de lidocaína isolada (L) e associada ao fentanil nas doses de 2,5, 5 e $7 \mathrm{mg} \mathrm{kg}^{-1}$ (F2,5, F5 e F7, respectivamente). Quinze minutos antes da punção peridural, todos os animais foram tranquilizados por via intravenosa (IV) com acepromazina $\left(0,05 \mathrm{mg} \mathrm{kg}^{-1}\right)$, além de um bolus (IV) de fentanil $\left(2,5 \mathrm{mg} \mathrm{kg}^{-1}\right)$, administrado imediatamente antes da injeção peridural. Foram avaliados: frequência cardíaca (FC), eletrocardiograma (ECG), pressão arterial sistólica (PAS), frequência respiratória ( $f$ ), gases sanguíneos, período de latência, duração e extensão do bloqueio sensitivo. Houve redução da FC após a anestesia peridural em relação ao basal em F2,5, F5 e F7. A FC foi superior no $L$ em relação ao $F 5$ e F7 aos 30 e 60 minutos após a anestesia peridural. O período de latência não variou entre os grupos, enquanto a duração do bloqueio foi superior no F5. Bloqueio sensitivo até a $6^{a}$ vértebra lombar foi observado em quatro animais no $L$. Bloqueio sensitivo até a $1^{a}$ vértebra lombar foi observado em cinco cães no F2,5 e F7 e em seis cães no F5. Conclui-se que a adição do fentanil à lidocaína determinou bloqueio sensitivo mais cranial em relação ao uso isolado desse anestésico local. Paralelamente, bloqueio anestésico mais duradouro foi determinado pela adição de $5 \mathrm{mg} \mathrm{kg}^{-1}$ de fentanil à lidocaína em relação aos demais tratamentos.

\begin{abstract}
The objective of this blind study was to investigate the cardiopulmonary and analgesic effects of different doses of fentanyl combined with lidocaine by epidural route in dogs. Twenty-eight dogs were distributed in four treatments: $5 \mathrm{mg}$ $\mathrm{kg}^{-1}$ of lidocaine with vasoconstrictor alone $(L)$ and in combination with 2.5, 5 e $7 \mathrm{mg} \mathrm{kg}^{-1}$ of fentanyl (F2.5, F5 e F7). The preanaesthetic medication was intravenous acepromazine $\left(0.05 \mathrm{mg} \mathrm{kg}^{-1}\right)$. An intravenous bolus of fentanyl (2.5 $\mathrm{mg} \mathrm{kg}^{-1}$ ) was administered immediately before epidural injection. Heart and respiratory rates, electrocardiography, systolic arterial blood pressure, rectal temperature, arterial blood gases, onset time, duration of anesthesia and sensitive block level were investigated. There was decrease of heart rate after epidural injection when compared with the baseline values in F2.5, F5 and F7 treatments. Heart rate was higher in $L$ when compared with F5 and F7 at 30 and 60 minutes after epidural anesthesia. Onset time was not statically different among the treatments. Duration of anesthesia was longer in F5 when compared with the other treatments. Sensitive block until the sixth lumbar vertebra occurred in four dogs in $L$. Sensitive block until the first lumbar vertebra occurred in five dogs in F2.5 and F7 and in six dogs in F5. In conclusion, the addition of fentanyl to lidocaine in epidural anesthesia allowed cranial sensory block in relation to the isolated use of lidocaine. The addition of fentanyl $\left(5 \mathrm{mg} \mathrm{kg}^{-1}\right)$ to lidocaine prolonged the anesthetic blockade when compared with others treatments.
\end{abstract}

Key words: opioid, local anesthetic, canine.

Palavras-chave: opioide, anestésico local, canina.

'Departamento de Cirurgia e Anestesiologia Veterinária, Faculdade de Ciências Agrárias, Universidade do Oeste Paulista (Unoeste), Rodovia Raposo Tavares, Km 572, Campus II, Bairro Limoeiro, 19067-175, Presidente Prudente, SP, Brasil. E-mail: renavarro@uol.com.br. *Autor para correspondência.

"Departamento de Clínica Médica de Pequenos Animais, Faculdade de Ciências Agrárias, Unoeste, Presidente Prudente, SP, Brasil. ${ }^{\text {IIIC } C u r s o ~ d e ~ M e d i c i n a ~ V e t e r i n a ́ r i a, ~ F a c u l d a d e ~ d e ~ C i e ̂ n c i a s ~ A g r a ́ r i a s, ~ U n o e s t e, ~ P r e s i d e n t e ~ P r u d e n t e, ~ S P, ~ B r a s i l . ~}$ 


\section{INTRODUÇÃo}

Na medicina veterinária, a anestesia peridural é normalmente empregada para realização de procedimentos cirúrgicos retroumbilicais, bem como adjuvante da anestesia geral para o controle da dor intra e pós-operatória. Em pequenos animais, os principais fármacos empregados nessa modalidade anestésica são a lidocaína, a bupivacaína e a ropivacaína (SKARDA \& TRANQUILLI, 2007), além de fármacos com potencial analgésico como cetamina (ISHIY et al., 2002), alfa ${ }_{2}$ adrenoceptores agonistas (GASPARINI et al., 2007) e opioides (CASSU et al., 2008; FREITAS et al., 2008; SILVA et al., 2008).

Estudos recentes têm demonstrado que o uso de opioides como adjuvantes da anestesia peridural permite a redução da dose dos anestésicos locais, além de conferir melhor qualidade analgésica e redução da duração do bloqueio motor (MARTINS et al., 2004; KANAI et al., 2007). Paralelamente, alguns estudos têm sugerido a possibilidade da extensão cranial do bloqueio anestésico, em função da adição de opioides aos anestésicos locais (AMINKOV, 1996; ISHIY et al., 2002; CASSU et al., 2008). A lidocaína, por exemplo, não é suficiente para produzir anestesia que viabilize a realização de cirurgias pré-umbilicais, quando empregada em

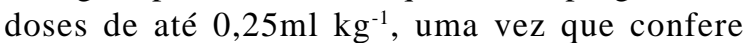
bloqueio sensitivo entre a quarta e a sexta vértebra lombar (CRUZ et al., 1997; GASPARINI et al., 2007). Adicionalmente, a administração peridural de anestésicos locais favorece o bloqueio simpático, podendo induzir hipotensão arterial e, em situações mais raras, parada cardíaca (SKARDA \& TRANQUILLI, 2007). Dessa forma, a adição dos opioides aos anestésicos locais é uma conduta favorável, com relatos promissores em relação à qualidade da anestesia e estabilidade cardiovascular (MARTINS et al., 2004; KANAI et al., 2007). Estudos realizados em cães demonstraram que a adição do fentanil à lidocaína promoveu extensão cranial do bloqueio sensitivo, permitindo a realização de ovariossalpingohisterectomia em cadelas, com mínimos efeitos depressores no sistema cardiovascular e respiratório (AMINKOV, 1996; CASSU et al., 2008).

Dessa forma, o presente estudo tem como objetivos investigar os efeitos cardiorrespiratórios e avaliar o bloqueio anestésico em função da administração da lidocaína com vasoconstrictor isolada e associada a diferentes doses de fentanil pela via peridural em cães.

\section{MATERIAL E MÉTODOS}

Foram avaliados 28 cães adultos, machos ou fêmeas, com peso médio de $7,9 \pm 4 \mathrm{~kg}$ e saudáveis. Estes são provenientes do canil da Instituição de Origem e foram selecionados por meio de exame físico e laboratorial (hemograma, dosagem sérica de ureia, creatinina, enzimas alanino aminotransferase, aspartato aminotransferase e fosfatase alcalina). Os animais foram submetidos a jejum alimentar e hídrico de 12 e três horas, respectivamente, antes da realização do experimento. Todos os cães receberam como medicação pré-anestésica (MPA) $0,05 \mathrm{mg} \mathrm{kg}^{-1}$ de maleato de acepromazina $0,2 \%^{\mathrm{a}}$ via intravenosa (IV). Transcorridos 15 minutos da MPA, a veia cefálica foi cateterizada, seguindo-se a administração de um bolus IV de fentanil ${ }^{\mathrm{b}}\left(2,5 \mathrm{mg} \mathrm{kg}^{-1}\right)$ a fim de aprofundar o grau de sedação proporcionado pela acepromazina, de modo a facilitar a administração via peridural, minimizando o desconforto do animal perante a injeção.

Um botão anestésico foi realizado no local da injeção, localizado mediante palpação das cristas ilíacas e da apófise espinhosa de L7. Os animais foram posicionados em decúbito esternal, estendendo-se os membros pélvicos cranialmente para punção do espaço peridural, cuja localização foi confirmada pela aspiração de uma gota do anestésico, depositada no canhão da agulha. A distribuição dos animais foi realizada ao acaso, em estudo cego, de modo que cada sete cães foram submetidos a um dos quatro tratamentos: $5 \mathrm{mg}$ $\mathrm{kg}^{-1}\left(0,25 \mathrm{ml} \mathrm{kg}^{-1}\right)$ de lidocaína $2 \%$ com vasoconstrictor (L) e associada a $2,5 \mathrm{mg} \mathrm{kg}^{-1}, 5 \mathrm{mg} \mathrm{kg} \mathrm{kg}^{-1}$ e $7 \mathrm{mg} \mathrm{kg}^{-1}$ de fentanil (F2,5, F5 e F7, respectivamente). A lidocaína foi associada ao fentanil, de modo a perfazer um volume final de $0,25 \mathrm{ml} \mathrm{kg}^{-1}$. A injeção dos fármacos foi realizada em período aproximado de 45 a 60 segundos, com velocidade constante.

Foram avaliados frequência cardíaca (FC) e ritmo cardíaco, mediante eletrocardiografia (ECG) ${ }^{\mathrm{d}}$; pressão arterial sistólica (PAS), mediante monitor de pressão arterial não invasivo ${ }^{\mathrm{e}}$, pela adaptação do manguito pediátrico na região proximal do rádio, respeitando-se uma relação de 0,4 entre a largura do manguito e o comprimento da circunferência do membro; frequência respiratória $(f)$, por meio da inspeção dos movimentos torácicos em um minuto; pressão parcial de gás carbônico $\left(\mathrm{PaCO}_{2}\right)$, pressão parcial de oxigênio $\left(\mathrm{PaO}_{2}\right)$, saturação de oxigênio na hemoglobina $\left(\mathrm{SaO}_{2}\right), \mathrm{pH}$, bicarbonato $\left(\mathrm{HCO}_{3}^{-}\right)$, mediante coleta de sangue por meio da punção da artéria femoral e avaliação dos gases sanguíneos ${ }^{\mathrm{f}}$; temperatura retal (T), com termômetro digital ${ }^{g}$. 
A avaliação dos gases sanguíneos foi feita antes da MPA, aos 30 e 60 minutos após a injeção peridural. Todos os outros parâmetros foram mensurados antes da MPA, 15 minutos após a MPA (anterior ao bolus de fentanil), cinco minutos e a cada 15 minutos após a anestesia peridural até o retorno do reflexo interdigital e movimento espontâneo do membro pélvico.

Foi determinado também o tempo entre a injeção peridural até o tempo para a perda do reflexo interdigital (período de latência), aferido por meio de estímulo nociceptivo mecânico, com pinça hemostática Kelly, realizado em intervalos de um minuto, até a ausência de reflexo interdigital em ambos os membros pélvicos. A partir daí, a anestesia foi avaliada com estímulos nociceptivos somáticos, aplicados a cada 15 minutos, imediatamente após a mensuração das variáveis paramétricas. A analgesia somática foi avaliada por meio da aplicação do teste do panículo, com pinça Kelly, na região dorsal, iniciando-se na área lombo-sacra, em direção cranial às vértebras lombares e torácicas, até ser identificada a resposta positiva do animal perante o estímulo nociceptivo. Essa avaliação permitiu a identificação da extensão do bloqueio anestésico, uma vez que os estímulos foram aplicados em áreas anatômicas específicas. Dessa forma, o estímulo foi aplicado em cada uma das vértebras, sendo registrado o ponto na coluna vertebral em que o animal apresentava sensibilidade (GASPARINI et al., 2007). A duração do bloqueio foi determinada pela aplicação de estímulo nociceptivo mecânico no espaço interdigital, com pinça hemostática Kelly, até o retorno da sensibilidade em ambos os membros pélvicos.

Foi utilizada análise de variância (ANOVA) para comparação entre os grupos, com contrastes verificados pelo teste de Tukey. Para comparar os momentos dentro de cada grupo foi utilizada análise de variância (ANOVA) para amostras relacionadas, com contrastes verificados pelo teste de Tukey. Adotou-se nível de significância de 5\%(ZAR, 1996).

\section{RESULTADOS}

Os quatro grupos foram comparáveis em relação aos dados demográficos, não sendo observadas diferenças significativas na idade e no peso dos animais avaliados. Aidade foi de $24 \pm 4,20 \pm 2,26 \pm 4$ e $22 \pm 5$ meses, com peso de $7 \pm 3,8 \pm 4,9 \pm 5$ e $7,5 \pm 4 \mathrm{~kg}$ para os tratamentos L, F2,5, F5 e F7, respectivamente. A distribuição em relação ao sexo dos animais foi aleatória.

Ocorreu redução significativa da FC após a MPA nos tratamentos F2,5 e F7 em relação aos valores basais. Comportamento semelhante foi observado aos cinco minutos após a injeção peridural em todos os animais tratados com fentanil (F2,5, F5 e F7), persistindo até 60 minutos. Na comparação entre grupos foram observados valores superiores de FC no tratamento L em relação ao F7 aos cinco, 30 e 60 minutos após a anestesia peridural, e em relação ao F5 aos 30 e 60 minutos (Tabela 1). Bradicardia foi observada em um animal do $\mathrm{L}$ aos cinco minutos, em dois animais do F2,5 entre cinco e 15 minutos após a anestesia peridural, em dois animais do F5 e em cinco animais do F7 a partir dos cinco minutos após a anestesia peridural, perdurando até 60 minutos.

Com relação às alterações de ritmo cardíaco, observou-se bloqueio átrio-ventricular (BAV) de primeiro grau em dois animais do $F 5$, aos 45 e 60 minutos após a anestesia peridural. Nos demais tratamentos, não foram identificadas alterações no ritmo cardíaco ao longo do período avaliado.

Houve redução significativa na pressão arterial sistólica (PAS) 15 minutos após a MPA no F2,5 e $\mathrm{L}$ em relação aos valores basais, persistindo até 30 e 60 minutos após a anestesia peridural, respectivamente. Em F5 e F7, redução significativa da PAS foi observada aos cinco e 15 minutos após a anestesia peridural em relação aos valores basais, respectivamente, persistindo até 60 minutos em ambos os tratamentos. Na comparação entre os tratamentos, não houve diferença significativa (Tabela 1).

Com relação às variáveis respiratórias e hemogasométricas, houve redução significativa na frequência respiratória em todos os momentos avaliados após a anestesia peridural em relação aos valores basais, sem diferença entre os tratamentos. Os animais tratados com lidocaína com vasoconstrictor (L) apresentaram valores inferiores de frequência respiratória 15 minutos após a MPA em relação aos demais tratamentos. Aumento significativo da $\mathrm{PaCO}_{2}$ foi observado aos 30 e 60 minutos após a administração da anestesia peridural em relação aos valores basais nos tratamentos F2,5 e F7 (Tabela1).

O período de latência foi de $4,8 \pm 1,5,4,0 \pm 0,8$,

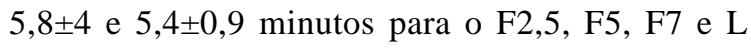
respectivamente, sem diferença significativa entre eles. A duração do bloqueio anestésico foi de $81 \pm 7,98 \pm 7$, $61 \pm 4$ e $69 \pm 13$ minutos para o F2,5, F5, F7 e L, respectivamente, sendo significativamente superior para o F5 (Tabela 2).

Houve dessensibilização ao teste do panículo até a $1^{\mathrm{a}}$ vértebra lombar (L1) em cinco animais no F2,5 e F7 e em seis animais no F5. Houve extensão do bloqueio anestésico, com propagação cranial às vértebras lombares em dois animais do F2,5 e em um 
Tabela 1 - Valores médios e desvio padrão da frequência cardíaca (FC), da pressão arterial sistólica (PAS), da frequência respiratória ( $f$ ), da pressão parcial de gás carbônico $\left(\mathrm{PaCO}_{2}\right)$, da pressão parcial de oxigênio $\left(\mathrm{PaO}_{2}\right)$, do $\mathrm{pH}$ e da saturação de oxigênio na hemoglobina $\left(\mathrm{SaO}_{2}\right)$ em cães sob anestesia peridural com lidocaína com vasoconstrictor isolada (L) e associada a $2,5 \mu \mathrm{g} \mathrm{kg}{ }^{-1}, 5 \mu \mathrm{g}$ $\mathrm{kg}^{-1}$ e $7 \mu \mathrm{kg}^{-1}$ de fentanil (F2,5, F5 e F7, respectivamente).

\begin{tabular}{|c|c|c|c|c|c|c|c|}
\hline & Antes da MPA & $\begin{array}{l}\text { 15’ após } \\
\text { MPA }\end{array}$ & $\begin{array}{l}\text { 5' após } \\
\text { epidural }\end{array}$ & $\begin{array}{l}\text { 15’ após } \\
\text { epidural }\end{array}$ & 30’ após epidural & $\begin{array}{l}\text { 45’ após } \\
\text { epidural }\end{array}$ & $\begin{array}{l}\text { 60' após } \\
\text { epidural }\end{array}$ \\
\hline \multicolumn{8}{|c|}{ FC (bat $\min ^{-1}$ ) } \\
\hline $\mathrm{L}$ & $112 \pm 28$ & $100 \pm 18$ & $84 \pm 22^{\dagger}$ & $89 \pm 25$ & $109 \pm 27^{\circ}$ & $99 \pm 24$ & $104 \pm 26^{\bullet}$ \\
\hline $\mathrm{F} 2,5$ & $100 \pm 12$ & $83 \pm 17 *$ & $64 \pm 9 *$ & $75 \pm 12 *$ & $74 \pm 9 *$ & $75 \pm 17 *$ & $81 \pm 12^{*}$ \\
\hline F5 & $90 \pm 10$ & $84 \pm 19$ & $64 \pm 11^{*}$ & $66 \pm 14 *$ & $65 \pm 19 *$ & $68 \pm 15^{*}$ & $69 \pm 19 *$ \\
\hline F7 & $101 \pm 19$ & $71 \pm 11^{*}$ & $54 \pm 14 *$ & $64 \pm 12 *$ & $63 \pm 17 *$ & $65 \pm 16^{*}$ & $67 \pm 17^{*}$ \\
\hline \multicolumn{8}{|c|}{ PAS (mmHg) } \\
\hline $\mathrm{L}$ & $131 \pm 9$ & $111 \pm 7^{*}$ & $100 \pm 10^{*}$ & $107 \pm 9 *$ & $110 \pm 11 *$ & $107 \pm 14^{*}$ & $105 \pm 14^{*}$ \\
\hline $\mathrm{F} 2,5$ & $129 \pm 19$ & $108 \pm 14 *$ & $108 \pm 11^{*}$ & $107 \pm 10 *$ & $110 \pm 12 *$ & $113 \pm 12$ & $117 \pm 24$ \\
\hline F5 & $122 \pm 7$ & $111 \pm 11$ & $114 \pm 14$ & $107 \pm 8^{*}$ & $107 \pm 12 *$ & $107 \pm 11^{*}$ & $107 \pm 14^{*}$ \\
\hline F7 & $127 \pm 7$ & $117 \pm 7$ & $109 \pm 10 *$ & $106 \pm 8^{*}$ & $108 \pm 13^{*}$ & $108 \pm 16^{*}$ & $116 \pm 17 *$ \\
\hline \multicolumn{8}{|c|}{$f\left(\operatorname{mov} \min ^{-1}\right)$} \\
\hline $\mathrm{L}$ & $24 \pm 7$ & $15 \pm 2 * \#$ & $14 \pm 2 *$ & $11 \pm 2 *$ & $16 \pm 3 *$ & $15 \pm 3^{*}$ & $15 \pm 3 *$ \\
\hline $\mathrm{F} 2,5$ & $28 \pm 6$ & $23 \pm 7$ & $16 \pm 5^{*}$ & $18 \pm 7^{*}$ & $20 \pm 5 *$ & $20 \pm 4 *$ & $20 \pm 6^{*}$ \\
\hline F5 & $26 \pm 6$ & $25 \pm 6$ & $20 \pm 7 *$ & $16 \pm 5^{*}$ & $18 \pm 6^{*}$ & $20 \pm 3 *$ & $21 \pm 4^{*}$ \\
\hline F7 & $32 \pm 7$ & $30 \pm 9$ & $18 \pm 6 *$ & $16 \pm 3^{*}$ & $18 \pm 5^{*}$ & $18 \pm 4 *$ & $24 \pm 2 *$ \\
\hline \multicolumn{8}{|c|}{$\begin{array}{l}\mathrm{PaCO}_{2} \\
\text { (mmHg) }\end{array}$} \\
\hline $\mathrm{L}$ & $31 \pm 4$ & & & & $34 \pm 4$ & & $34 \pm 1$ \\
\hline $\mathrm{F} 2,5$ & $33 \pm 3$ & & & & $38 \pm 4 *$ & & $37 \pm 3^{*}$ \\
\hline F5 & $34 \pm 4$ & & & & $37 \pm 1$ & & $38 \pm 3$ \\
\hline F7 & $32 \pm 2$ & & & & $37 \pm 2 *$ & & $34 \pm 3 *$ \\
\hline \multicolumn{8}{|c|}{$\mathrm{PaO}_{2}(\mathrm{mmHg})$} \\
\hline $\mathrm{L}$ & $99 \pm 10$ & & & & $100 \pm 15$ & & $101 \pm 15$ \\
\hline $\mathrm{F} 2,5$ & $102 \pm 14$ & & & & $101 \pm 15$ & & $99 \pm 17$ \\
\hline F5 & $100 \pm 17$ & & & & $102 \pm 17$ & & $99 \pm 11$ \\
\hline F7 & $90 \pm 5$ & & & & $101 \pm 7$ & & $100 \pm 3$ \\
\hline \multicolumn{8}{|l|}{$\mathrm{pH}$} \\
\hline $\mathrm{L}$ & $7,41 \pm 0,02$ & & & & $7,39 \pm 0,03 ?$ & & $7,39 \pm 0,01$ \\
\hline $\mathrm{F} 2,5$ & $7,31 \pm 0,1^{\#}$ & & & & $7,30 \pm 0,01$ & & $7,31 \pm 0,01^{\#}$ \\
\hline F5 & $7,33 \pm 0,1$ & & & & $7,30 \pm 0,01$ & & $7,35 \pm 0,1$ \\
\hline F7 & $7,34 \pm 0,02$ & & & & $7,33 \pm 0,1$ & & $7,32 \pm 0,02$ \\
\hline \multicolumn{8}{|c|}{$\mathrm{SatO}_{2}(\%)$} \\
\hline $\mathrm{L}$ & & $99 \pm 0,7$ & & & $98 \pm 0,5$ & & $99 \pm 0,8$ \\
\hline $\mathrm{F} 2,5$ & & $98 \pm 0,5$ & & & $98 \pm 0,8$ & & $98 \pm 0,7$ \\
\hline F5 & & $97 \pm 0,5$ & & & $98 \pm 1$ & & $97 \pm 1,5$ \\
\hline F7 & & $98 \pm 0,5$ & & & $98 \pm 0,8$ & & $98 \pm 0,7$ \\
\hline
\end{tabular}

*diferença significativa em relação aos valores basais, $\mathrm{P}<0,05 .{ }^{\dagger}$ diferença significativa entre $\mathrm{L}$ e $\mathrm{F} 7, \mathrm{P}<0,05$. ${ }^{\circ}$ diferença significativa de $\mathrm{L}$ em F5 e F7, P<0,05. "diferença significativa entre F2,5 e L, P<0,05. ?diferença significativa de L em relação ao F2,5 e F5, P<0,05.

animal do F5, cujo bloqueio atingiu até a décima terceira vértebra torácica (T13) e em dois animais do F7, cujo bloqueio atingiu até a décima segunda vértebra torácica (T12) e décima vértebra torácica (T10) (Tabela 2).

\section{DISCUSSÃO}

A frequência cardíaca (FC) declinou após a administração da acepromazina em relação aos valores basais nos tratamentos F2,5 e F7, provavelmente em função do efeito ansiolítico determinado por esse fenotiazínico (FANTONI \& CORTOPASSI, 2008), porém os valores observados mantiveram-se dentro dos limites fisiológicos para a espécie. Todavia, após a anestesia peridural, observou-se redução significativa da FC nos tratamentos F2,5, F5 e F7, sugerindo que o fentanil determinou um incremento na depressão dessa variável. Em cães, após a administração de $10 \mathrm{mg} \mathrm{kg}^{-1}$ 
Tabela 2 - Valores médios e desvio padrão do período de latência (minutos), da duração do bloqueio anestésico (minutos) e da extensão do bloqueio sensitivo em cães sob anestesia peridural com lidocaína com vasoconstrictor isolada (L) e associada a $2,5 \mu \mathrm{g} \mathrm{kg}^{-1}$, $5 \mu \mathrm{g} \mathrm{kg}{ }^{-1}$ e $7 \mu \mathrm{kg}^{-1}$ de fentanil (F2,5, F5 e F7, respectivamente).

\begin{tabular}{|c|c|c|c|c|}
\hline & $\mathrm{L}$ & $\mathrm{F} 2,5$ & F5 & F7 \\
\hline Latência & $5,4 \pm 0,9$ & $4,8 \pm 1,5$ & $4 \pm 0,8$ & $5,8 \pm 4$ \\
\hline Duração do bloqueio & $69 \pm 13$ & $81 \pm 7$ & $98 \pm 7 \#$ & $61 \pm 4$ \\
\hline \multirow{3}{*}{$\begin{array}{l}\text { Extensão máxima } \\
\text { bloqueio }\end{array}$} & L6 (n=4) & L1 (n=5) & L1 $(n=6)$ & L1 (n=5) \\
\hline & L5 (n=2) & $\mathrm{T} 13(\mathrm{n}=2)$ & $\mathrm{T} 13(\mathrm{n}=1)$ & $\mathrm{T} 12(\mathrm{n}=1)$ \\
\hline & L4 (n=1) & & & $\mathrm{T} 10(\mathrm{n}=1)$ \\
\hline
\end{tabular}

\#diferença significativa de F5 em relação aos demais tratamentos. P<0,05.

de fentanil IV, concentração plasmática de $5 \mathrm{ng} \mathrm{ml}^{-1}$ foi alcançada após 2,5 minutos, declinando aos 20 minutos após a administração (SANO et al., 2006). Dessa forma, no presente estudo, a redução da FC é atribuída não apenas à administração peridural do fentanil, mas também ao efeito somatório resultante da administração intravenosa, justificando a menor depressão desse parâmetro nos animais do tratamento L, que não receberam fentanil via peridural. A FC é controlada essencialmente por um componente que se origina de neurônios cardíacos vagais parassimpáticos no núcleo ambíguo, situado na medula, que fazem sinapse com gânglios localizados na base do coração. O fentanil reduz a frequência e a amplitude da transmissão GABAérgica para esses neurônios cardíacos vagais, culminando com a redução da FC (GRIFFIOEN et al., 2004). No entanto, embora a FC tenha sofrido redução após todos os tratamentos com fentanil, a ocorrência de bradicardia FC $<60$ bat min $^{-1}$ (HASKINS, 2007) foi mais pronunciada nos animais do F7, sugerindo um efeito cronotrópico negativo dose-dependente.

Além da redução da FC, o fentanil é capaz de produzir uma variedade de arritmias em razão do aumento da atividade vagal, como BAV de primeiro e segundo graus (SANTOS et al., 2001). No entanto, no atual estudo, observou-se discreta ocorrência de arritmias, sendo diagnosticado BAV de primeiro grau em apenas dois animais do F5. Todavia, esse ritmo não está associado às manifestações clínicas, sendo considerado um achado que não requer tratamento.

A redução da PAS foi observada em todos os grupos após a MPA, embora diferença significativa tenha sido detectada apenas nos tratamentos F2,5 e L em relação aos valores basais. Esse resultado justificase pela ação da acepromazina, que determina bloqueio dos receptores $\alpha_{1}$ adrenérgicos periféricos, induzindo vasodilatação e redução da pressão arterial (FANTONI \& CORTOPASSI, 2008). Após a anestesia peridural, a PAS manteve-se inferior em relação aos valores basais em todos os tratamentos, porém não se observou hipotensão (PAS<100mmHg) (HASKINS, 2007), corroborando resultados prévios que demonstraram estabilidade da pressão arterial após administração de lidocaína com vasoconstrictor isolada ou associada ao fentanil pela via peridural (NOLTE et al., 1983; AMINKOV, 1996; CASSU et al., 2008).

Adicionalmente, as variáveis respiratórias mantiveram-se estáveis, observando-se discreta redução na frequência respiratória em relação aos valores basais, após a anestesia peridural, com consequente incremento da $\mathrm{PaCO}_{2}$ em todos os tratamentos, porém os valores observados mantiveramse dentro dos limites fisiológicos para a espécie, corroborando resultados prévios (NOLTE et al., 1983; AMINKOV, 1996; CASSU et al., 2008). Valores inferiores de $\mathrm{pH}$ foram detectados nos animais tratados com fentanil em relação ao tratamento com lidocaína isolada, sobretudo no F2,5, com observação de discreta acidose respiratória $(\mathrm{pH}<7,35)$, porém esse achado não teve implicações clínicas.

Alguns autores têm confirmado a redução do período de latência do anestésico local em função da adição do fentanil no espaço peridural (JOHNSON et al., 1991; AMINKOV, 1996). Porém, no presente estudo, o período de latência não diferiu entre os tratamentos, concordando com resultados relatados no homem e no cão, nos quais a adição do fentanil não reduziu o tempo de latência do anestésico local (MARTINS et al., 2004; CASSU et al. 2008; SILVA et al., 2008).

Em relação à duração do bloqueio anestésico, a adição de $5 \mathrm{mg} \mathrm{kg}^{-1}$ de fentanil à lidocaína com vasoconstrictor resultou em bloqueio sensitivo

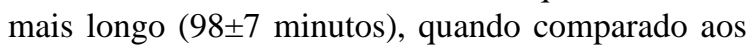
demais tratamentos. A duração do bloqueio anestésico pode variar em função da dose do fármaco, bem como do volume administrado (SKARDA \& TRANQUILLI, 2007). No atual estudo, independente da dose empregada, o volume não variou entre os tratamentos, sendo preconizado o volume final de $0,25 \mathrm{ml} \mathrm{kg}^{-1}$, 
conforme descrito por outros autores (ISHIY et al., 2002; GASPARINI et al., 2007; FREITAS et al., 2008). Dessa forma, os resultados sugerem que o incremento da dose do fentanil de 2,5 para $5 \mathrm{mg} \mathrm{kg}^{-1}$ possibilitou maior duração do bloqueio. Em estudo semelhante, a duração do bloqueio anestésico conferido pela adição de $4 \mathrm{mg}$ $\mathrm{kg}^{-1}$ de fentanil à lidocaína com vasoconstrictor foi de 84 $\pm 7,8$ minutos (FREITAS et al., 2008), sendo esse resultado próximo à duração do bloqueio observado no atual estudo no F2,5, que foi de $81 \pm 7$ minutos. No entanto, no F7, embora maior dose de fentanil tenha sido utilizada, a duração do bloqueio anestésico foi inferior, provavelmente em virtude da menor dose de lidocaína empregada, visando a manter o mesmo volume final $\left(0,25 \mathrm{ml} \mathrm{kg}^{-1}\right)$. Considerando-se o peso médio de $8 \mathrm{~kg}$, o volume de lidocaína empregado foi de $1,6 \mathrm{ml}$, $1,2 \mathrm{ml}$ e $0,88 \mathrm{ml}$ nos tratamentos $\mathrm{F} 2,5$, F5 e F7, respectivamente.

Todavia, apesar do incremento da duração do bloqueio anestésico no F5, esse efeito foi pouco pronunciado, visto que o uso isolado da lidocaína com vasoconstrictor no espaço peridural de cães promove bloqueio anestésico entre 60 e 120 minutos (SKARDA \& TRANQUILLI, 2007). No atual estudo, o bloqueio anestésico foi prolongado no máximo em 29 minutos pela adição de $5 \mathrm{mg} \mathrm{kg}^{-1}$ de fentanil em relação ao uso isolado da lidocaína. A curta duração de ação do fentanil justifica-se em função de sua alta lipossolubilidade, a qual permite que o fármaco atravesse rapidamente as meninges, atingindo o líquido cefalorraquidiano (LCR) em poucos minutos, de modo a resultar em curto período de latência e de ação (SKARDA \& TRANQUILLI, 2007). No homem, após administração peridural de fentanil a concentração máxima desse opioide no LCR foi atingida entre 10-20 minutos, começando a declinar aos 45 minutos (HARUKUNI et al., 1995), divergindo dos resultados obtidos com opioides hidrossolúveis, como a morfina, cuja latência foi de 20 a 60 minutos, com efeito analgésico de 16 a 24 horas no cão (VALVERDE et al., 1992).

Estudos recentes têm demonstrado que a extensão máxima do bloqueio sensitivo determinado pela administração peridural lombo-sacra de $0,25 \mathrm{ml} \mathrm{kg}$ ${ }^{1}$ de lidocaína a $2 \%$ em cães não ultrapassa a quarta vértebra lombar (ISHIY et al., 2002; GASPARINI et al., 2007), concordando com os resultados observados no presente estudo, em que a altura máxima do bloqueio anestésico foi até a quarta vértebra lombar, sendo mais frequente somente até a sexta vértebra lombar no tratamento L. Paralelamente, no atual estudo, observouse ausência de sensibilidade até a primeira vértebra lombar em todos os animais tratados com fentanil associado à lidocaína com vasoconstrictor. Isso sugere que a adição do fentanil alterou o nível do bloqueio sensitivo em relação ao bloqueio determinado pela lidocaína com vasoconstrictor, permitindo dessensibilização mais cranial em relação ao uso isolado desse anestésico local e concordando com resultados prévios relatados no cão (AMINKOV, 2006; CASSU et al., 2008).

\section{CONCLUSÕES}

Conclui-se que a adição de diferentes doses de fentanil à lidocaína com vasoconstrictor determinou bloqueio sensitivo mais cranial em relação ao uso isolado desse anestésico local. Paralelamente, a dose de $5 \mathrm{mg} \mathrm{kg}^{-1}$ de fentanil aumentou a duração do bloqueio anestésico em relação aos demais tratamentos. Mínimas alterações foram observadas em relação à pressão arterial sistólica e às variáveis respiratórias; porém, a dose de $7 \mathrm{mg} \mathrm{kg}^{-1}$ de fentanil induziu bradicardia com maior frequência em relação aos demais tratamentos.

\section{FONTES DE AQUISIÇÃO}

a - Acepran 0,2\%, Univet, São Paulo, Brasil.

b - Fentanest, Cristália, Itapira, SP., Brasil.

c - Xislestesin 2\% com vasoconstrictor, Cristália, Itapira, SP., Brasil.

d - Eletrocardiógrafo CARDIOTEST EK 5, São Paulo, Brasil. e - Doppler 841-A, Parks Medical Electronics, Las Vegas, EUA f - 248-pH Blood Gas Analyser, Ciba Corning Diagnostics, Medfield, MA, USA.

g - Termômetro digital, BD, São Paulo, Brasil.

\section{COMITÊ DE ÉTICA}

Este estudo foi aprovado pelo Comitê de Ética em Pesquisa (CEP) da Universidade do Oeste Paulista (protocolo n.134/03) e realizado de acordo com os princípios éticos na experimentação animal.

\section{REFERÊNCIAS}

AMINKOV, B.Y. Comparison between lidocaine alone and fentanyl with lidocaine for epidural anaesthesia in dogs. Revista de Medicina Veterinaria, v.147, 819-824, 1996.

CASSU, R.N. et al. Anestesia epidural com lidocaína isolada e associada ao fentanil para realização de ovário-salpingohisterectomia em cadelas. Arquivo Brasileiro de Medicina Veterinária e Zootecnia, v.60, p.825-831, 2008. Disponível em: http://www.scielo.br/scielo. Acesso em: 15 dez. 2008. doi: 10.1590/S0102-09352008000400008.

CRUZ, M.L. et al. Epidural anaesthesia using lignocaine, bupivacaine or a mixture of lignocaine and bupivacaine in dogs. Journal of Veterinary Anaesthesia, v.24, p.30-33, 1997. 
FANTONI, D.T.; CORTOPASSI, S.R.G. Tranqüilizantes e sedativos. In: ANDRADE, S.F. Manual de terapêutica veterinária. 3.ed. São Paulo: Roca, 2008. p.466-469.

FREITAS, G.C. et al. Analgesia trans e pós-operatória da morfina ou fentanil por via epidural em cães submetidos à biópsia atlantoaxial. ARS Veterinária, v.24, p.103-109, 2008. Disponível em: www.arsveterinaria.org.br/index.php/ars/.../178. Acesso em: 17 ago. 2009.

GASPARINI, S.S. et al. Anestesia epidural com ropivacaína, lidocaína ou associação de lidocaína e xilazina em cães. Efeitos cardiorrespiratório e analgésico. Ciência Rural, v.37, p.418424, 2007. Disponível em: http://www.scielo.br/scielo. Acesso em: 12 dez. 2008. doi: 10.1590/S0103-84782007000200019.

GRIFFIOEN, K.J.S. et al. Fentanyl inhibits GABAergic neurotransmission to cardiac vagal neurons in the nucleus ambiguus. Brain Research, v.1007, p.109-115, 2004. Disponível em: http://www.ncbi.nlm.nih.gov/sites/ entrez?cmd=search\&db=pubmed. Acesso em: 08 mar. 2009.

HARUKUNI, I. et al. The comparison of epidural fentanyl,epidural lidocaine, and intravenous fentanyl in patients undergoing gastrectomy. Anesthesia and Analgesia, v.81, p. 1169-1174, 1995.

HASKINS, S.C. Monitoring anesthetized patients. In: TRANQUILLI, W.J. et al. Lumb \& Jones' veterinary anesthesia and analgesia. 4.ed. Oxford: Blackwell, 2007. Cap.19, p.533-560.

ISHIY, H. M. et al. Uso da lidocaína isolada ou associada à quetamina ou ao butorfanol, em anestesia epidural em cadelas submetidas à ovariosalpingohisterectomia. Revista Brasileira de Ciência Veterinária, v.9, p.134-136, 2002.

JOHNSON, C. et al. Comparison of onset time between $0.5 \%$ bupivacaine and 3\% 2-chloroprocaine with and without 75 micrograms fentanyl. Regional Anesthesia, v.16, p.228231, 1991.

KANAI, A. et al. Regression of sensory and motor blockade, and analgesia during continuous epidural infusion of ropivacaine and fentanyl in comparison with other local anesthetics. Pain Medicine, v.8, p.546-553, 2007. Disponível em: http:// www.ncbi.nlm.nih.gov/sites/entrez?cmd=search\&db=pubmed.
Acesso em: 16 jun. 2009. doi:10.1111/j.15264637.2006.00174.x.

MARTINS, C.A. et al. Epidural caudal block: evaluation of length of analgesia with the association of lidocaine, fentanyl and clonidine. Revista Brasileira de Anestesiologia, v.54, p.501-505, 2004. Disponível em: http://www.scielo.br/ scielo.php?script=sci_serial\&pid=0034-7094. Acesso em: 11 mai. 2009. doi: 10.1590/S0034-70942004000400006.

NOLTE, I. et al. Cardiovascular effects of epidural blocks in dogs. Journal of Small Animal Practice, v.24, p.17-21, 1983.

SANO, T. et al. Pharmacokinetics of fentanyl after single intravenous injection and constant rate infusion in dogs. Veterinary Anaesthesia and Analgesia, v.33, p.266-273, 2006. Disponível em: http://www.ncbi.nlm.nih.gov/sites/ entrez?cmd=search\&db=pubmed. Acesso em: 28 mai. 2009. doi:10.1111/j.1467-2995.2005.00266.x.

SANTOS, P.S.P. et al. Eletrocardiografia de cães submetidos a diferentes concentrações de desflurano, pré-tratados ou não com a associação de fentanil/ droperidol. Ciência Rural, v.31, p.805-811, 2001. Disponível em: http://www.scielo.br/ scielo. Acesso em: 10 mai. 2009. doi: 10.1590/S010384782001000500011 .

SILVA, B.M. et al. Ropivacaína isolada e associada ao fentanil ou ao tramadol administrados pela via peridural de cães. Ciência Rural, v.38, p.2197-2202, 2008. Disponível em: http:// www.scielo.br/scielo.php?script=sciarttext \&pid= S010384782008000800002\&Ing=pt\&nrm=iso. Acesso em: 11 jun. 2009. doi: 10.1590/S0103-84782008000800017.

SKARDA, R.T.; TRANQUILLI, W.J. Local anesthetics. In: TRANQUILLI, W.J. et al. Lumb \& Jones' veterinary anesthesia and analgesia. 4.ed. Oxford: Blackwell, 2007. Cap.14, p.395-418.

VALVERDE, A. et al. Cisternal CSF and serum concentrations of morphine following epidural administration in the dog. Journal of Veterinary Pharmacology and Therapeutics, v.15, p.91-95, 1992.

ZAR, J.H. Biostatistical analysis. New Jersey: PrenticeHall Upper Saddle River, 1996. p.718. 